\title{
Cost-effectiveness of pulmonary resection and systemic chemotherapy in the management of metastatic soft tissue sarcoma: A combined analysis from the University of Texas M. D. Anderson and Memorial Sloan-Kettering Cancer Centers
}

Geoffrey A. Porter, MD

Scott B. Cantor, $\mathrm{PhD}^{\mathrm{b}}$

Garrett L. Walsh, MD ${ }^{\mathrm{b}}$

Valerie W. Rusch, MD ${ }^{\mathrm{c}}$

Dennis H. Leung, $\mathrm{PhD}^{\mathrm{d}}$

Alma Y. DeJesus, MSN ${ }^{\mathrm{b}}$

Raphael E. Pollock, MD, PhD ${ }^{\mathrm{b}}$

Murray F. Brennan, MD ${ }^{\mathrm{C}}$

Peter W. T. Pisters, MD
From Dalhousie University, Halifax, Nova Scotia, Canada, ${ }^{a}$ The University of Texas M. D. Anderson Cancer Center, Houston, Tex, ${ }^{\text {b }}$ Memorial Sloan-Kettering Cancer Center, New York, NY, and The University of Singapore, Singapore. ${ }^{\mathrm{d}}$

Received for publication Sept 23, 2003; revisions requested Nov 13, 2003; revisions received Nov 20, 2003; accepted for publication Nov 20, 2003.

Address for reprints: Geoffrey A. Porter, MD, Department of Surgery, 7-007 QEII Health Sciences Center, Dalhousie University, 1278 Tower Rd, Halifax, Nova Scotia, Canada B3H 2Y9 (E-mail: Geoff.Porter @dal.ca).

J Thorac Cardiovasc Surg 2004;127:1366-72 $0022-5223 / \$ 30.00$

Copyright () 2004 by The American Association for Thoracic Surgery

doi:10.1016/j.jtcvs.2003.11.016
Background: We sought to determine the cost-effectiveness of different treatment strategies for patients with pulmonary metastases from soft tissue sarcoma.

Methods: We constructed a decision tree to model the outcomes of 4 treatment strategies for patients with pulmonary metastases from soft tissue sarcoma: pulmonary resection, systemic chemotherapy, pulmonary resection and systemic chemotherapy, and no treatment. Data from 1124 patients with pulmonary metastases from soft tissue sarcoma were used to estimate disease-specific survival for pulmonary resection and no treatment. Outcomes of systemic chemotherapy and pulmonary resection and of systemic chemotherapy were estimated by assuming a 12-month improvement in disease-specific survival with chemotherapy; this was done on the basis of the widely held but unproven assumption that chemotherapy provides a survival benefit in patients with metastatic soft tissue sarcoma. Direct costs were examined for a series of patients who underwent protocol-based pulmonary resection or doxorubicin/ifosfamide-based chemotherapy.

Results: The mean cost of pulmonary resection was $\$ 20,339$ per patient; the mean cost of 6 cycles of chemotherapy was $\$ 99,033$. Compared with no treatment and assuming a 12-month survival advantage with chemotherapy, the incremental costeffectiveness ratio was $\$ 14,357$ per life-year gained for pulmonary resection, $\$ 104,210$ per life-year gained for systemic chemotherapy, and $\$ 51,159$ per life-year gained for pulmonary resection and systemic chemotherapy. Compared with pulmonary resection, the incremental cost-effectiveness ratio of pulmonary resection and systemic chemotherapy was $\$ 108,036$ per life-year gained. Sensitivity analyses showed that certain patient and tumor features, as well as the assumed benefit of chemotherapy, affected cost-effectiveness.

Conclusions: For patients with pulmonary metastases from soft tissue sarcoma who were surgical candidates, pulmonary resection was the most cost-effective treatment strategy evaluated. Even with favorable assumptions regarding its clinical benefit, systemic chemotherapy alone, compared with no treatment, was not a cost-effective treatment strategy for these patients. 


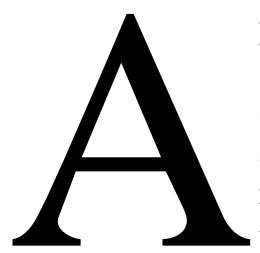

pproximately 8300 new cases of soft tissue sarcoma (STS) are diagnosed annually in the United States, and fewer than $50 \%$ of patients with this diagnosis will receive curative treatment with current treatment modalities. ${ }^{1}$ The lungs are the most common site of metastatic disease; pulmonary metastases affect $20 \%$ to $38 \%$ of all STS patients. ${ }^{2-4}$ Indeed, most patients who die of STS will have pulmonary metastases.

Several studies have demonstrated that a subgroup of patients with isolated, resectable pulmonary metastases from STS may be cured by pulmonary metastasectomy. ${ }^{3-11}$ These studies show pulmonary resection (PR) to be associated with low perioperative mortality rates, actuarial 3-year survival of $20 \%$ to $54 \%$, and 5-year survival of $21 \%$ to $51 \%$. However, some of these studies are limited by median follow-up durations of less than 3 years. ${ }^{6,7}$ Nevertheless, PR, when possible, has been advocated as the only potentially curative treatment in STS patients with lung metastases. ${ }^{12}$

Anthracycline-based chemotherapy is commonly used in patients with metastatic STS, ${ }^{13,14}$ although there has never been a randomized clinical trial comparing systemic chemotherapy with best supportive care in this group of patients. The combination of systemic chemotherapy and resection of STS pulmonary metastases is also commonly used. ${ }^{15-17}$ This approach is believed by some physicians to allow for more complete treatment of both macroscopic and occult microscopic metastatic disease. Unfortunately, a randomized trial designed to evaluate the potential clinical benefit of metastasectomy plus chemotherapy (European Organization for Research and Treatment of Cancer trial 62933) closed because of poor accrual. As a consequence, there is no consensus on the optimal therapeutic approach for patients with pulmonary metastases from STS.

In the absence of definitive clinical trials to guide treatment decisions, other considerations enter into decisionmaking for patients with metastatic STS. These considerations include the natural history of the disease, toxicities of currently available treatments, and, increasingly, issues of cost-effectiveness. The objective of this study was to examine the cost-effectiveness of different treatment options for patients with pulmonary metastases from STS.

\section{Patients and Methods}

\section{Decision Analysis}

Using a decision-analytic approach, ${ }^{18,19}$ we modeled the outcomes of 4 strategies for the treatment of STS pulmonary metastases: PR, systemic chemotherapy, PR and systemic chemotherapy $(\mathrm{PR}+\mathrm{C})$, and no treatment (NoRx). Consistent with the methodology of decision analysis, we estimated the expected costs and expected outcomes for each strategy. A summary of this model is depicted in Figure 1.

\section{Clinical Outcomes}

Median disease-specific survival was used as the primary outcome measure. This was estimated for the PR and NoRx groups on the basis of an updated outcome analysis of 1124 consecutive patients with STS pulmonary metastases treated and followed up at Memorial Sloan-Kettering Cancer Center. Data from the 235 patients within this cohort who underwent complete resection of all pulmonary metastases were used to estimate the outcome for PR; data from the remaining 889 patients were used to estimate the outcome for NoRx. This single-institution series represents a prospective cohort of patients with STS pulmonary metastases. ${ }^{4}$ The treatment groups (PR or NoRx) were not randomized in this cohort, thus creating potential patient- and disease-related biases. However, as a consecutive series (1982-1997), these data are believed to be the most comprehensive currently available outcome data for patients with STS pulmonary metastases. Sensitivity analyses were performed to examine the effect of any imprecision in the outcomes estimated for these 2 groups.

Outcomes of systemic chemotherapy and PR $+\mathrm{C}$ were estimated by generously assuming a 12-month improvement in disease-specific survival with chemotherapy in the base case (NoRx and PR, respectively). This was done on the basis of the widely held but unproven assumption that chemotherapy provides a survival benefit in patients with metastatic STS. All survival outcomes were discounted at a rate of $3 \%$ per annum and were calculated on a yearly basis.

\section{Costs}

Costs-not charges - were used in all economic analyses. Total costs were calculated as the sum of fixed and variable direct costs. Variable costs, which represent costs that are saved if a given procedure is not performed (eg, the cost of a radiologist's interpreting a chest computed tomography study), were determined with the aid of specialized computer software (Hospital Cost Consultants, Chicago, IL) and relied on information from a general ledger. Included in these variable costs were professional costs incurred by the hospital. Fixed costs, which represent costs that are incurred even if a procedure is not performed (eg, the overhead costs associated with a computed tomography scanner), were obtained by means of a statistical allocation from The University of Texas M. D. Anderson Cancer Center's cost accounting software. Total costs did not include any measurement or estimation of costs of patient time, non-health-care costs (eg, baby-sitting or transportation), or lost wages. All cost data were indexed to 2001 US dollars by multiplying recorded costs by the percentage inflationary increase, based on the medical component of the consumer price index, for each fiscal year in a compound manner.

Costs of chemotherapy were obtained from a cohort of 26 patients with metastatic STS who received contemporary STS chemotherapy consisting of doxorubicin $\left(75 \mathrm{mg} / \mathrm{m}^{2}\right.$ per cycle) and ifosfamide ( $10 \mathrm{~g} / \mathrm{m}^{2}$ per cycle). Chemotherapy was provided in the context of a standardized treatment protocol at The University of Texas M. D. Anderson Cancer Center. Patients received a median of 4 cycles (range, 2-7) of chemotherapy. We included costs associated with in-hospital and outpatient care from the first day of chemotherapy to 30 days after completion of the last cycle. The mean cost per cycle of chemotherapy was calculated, and we then 


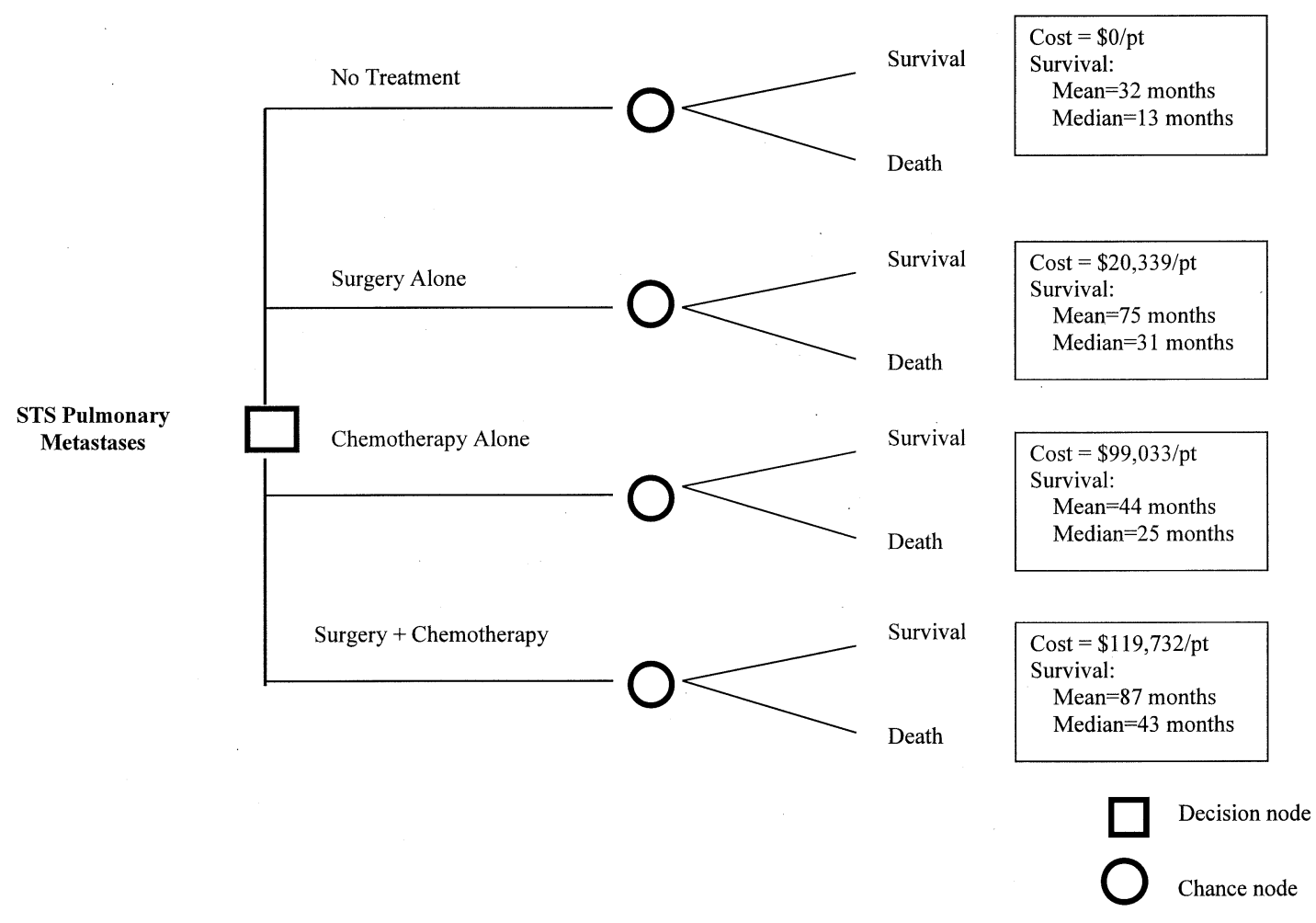

Figure 1. Decision tree. Summary of the analytic model comparing 4 hypothetical management strategies for patients with soft tissue sarcoma pulmonary metastases. The algorithm models possible outcomes of no treatment, pulmonary resection alone, systemic chemotherapy alone, and pulmonary resection and systemic chemotherapy. Summary costs and outcomes (prior to discounting) are included at the right of the figure. pt, Patient.

assumed that patients received 6 cycles of chemotherapy for the systemic chemotherapy and $\mathrm{PR}+\mathrm{C}$ base model.

Costs associated with surgery were obtained from a consecutive series of 46 patients who underwent resection of STS pulmonary metastases at M. D. Anderson Cancer Center. Pulmonary metastasectomy was performed according to an institutional clinical pathway protocol. Operative approaches included posterolateral thoracotomy, median sternotomy, clamshell (bilateral anterolateral) thoracotomy, and staged bilateral posterolateral thoracotomies. Additional resectable pulmonary metastases developed in 6 patients within 1 year of initial PR and were treated with repeat resection. Costs included the preoperative consultations, all inpatient care, and all outpatient care up to 30 days after discharge. Costs of repeat and staged resections were added to the costs of the original operation for each patient thus treated. All surgery costs were expressed on a per-patient basis.

For this study, we assumed that the costs of NoRx were the baseline costs of supportive care and were applicable to all 4 groups; therefore, these costs were not estimated or calculated. The costs of $\mathrm{PR}+\mathrm{C}$ were calculated by simply adding the costs calculated for PR alone and systemic chemotherapy alone. Because all examined costs occurred within the first year, no cost discounting was performed.

The measure of cost-effectiveness was the incremental costeffectiveness ratio (ICER), which is the difference in the costs of 2 strategies divided by the difference in survival with the 2 strategies. Otherwise stated, the ICER expressed the additional cost per life-year gained of one treatment strategy versus another. The ICER is a widely used, standard measure of cost-effectiveness. $^{20,21}$ In this study, the ICER was calculated as follows:

$$
\left.\operatorname{ICER}=\left[C_{x}-C_{y} / S_{x}-S_{y}\right)\right] / 12,
$$

where $C_{x}$ is the cost per patient of treatment strategy $x, C_{Y}$ is the cost per patient of treatment strategy $y, S_{x}$ is the survival (in months) with treatment strategy $x$, and $S_{y}$ is the survival (in months) with treatment strategy $y$.

Because no appropriate published data exist that quantify the survival benefit associated with chemotherapy in STS patients with pulmonary metastases, we performed a 1-way sensitivity analysis by altering the assumed benefit of chemotherapy in the systemic chemotherapy and $\mathrm{PR}+\mathrm{C}$ groups. Sensitivity analyses were also performed for specific patient subgroups (by age, grade, anatomic site, and disease-free interval) with the assumption that costs did not differ across these subgroups but that outcomes did. We also examined the effect on the ICER of delivering 4 cycles of chemotherapy instead of 6 .

\section{Results}

Costs and survival of the 4 strategies, in the base case model and before discounting, are presented in Figure 1. The mean cost of PR was \$20,339 (SEM, \$2718); the mean cost of 


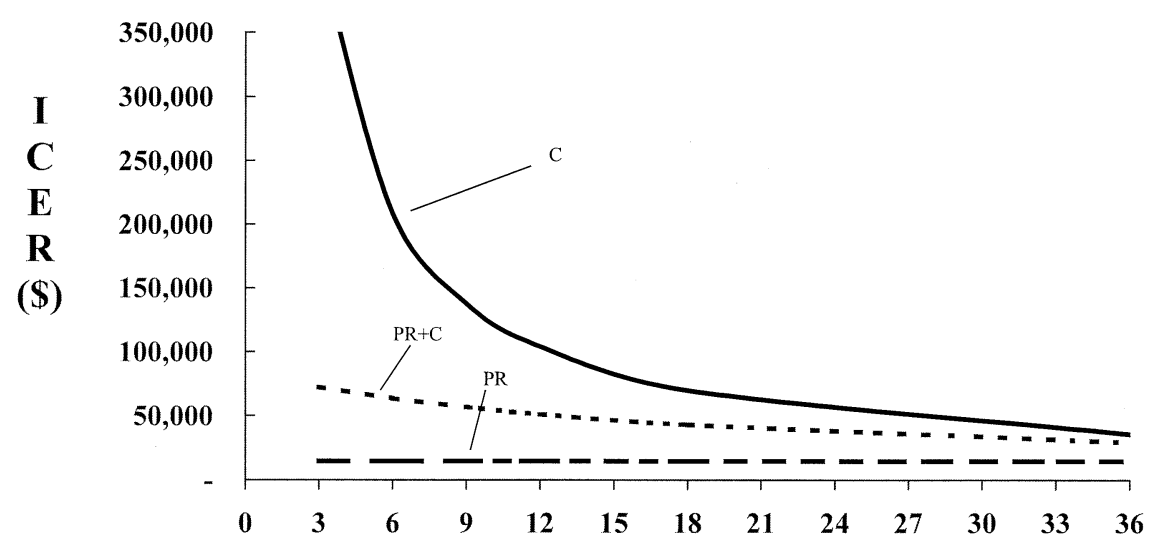

A

Assumed chemotherapy benefit (months)

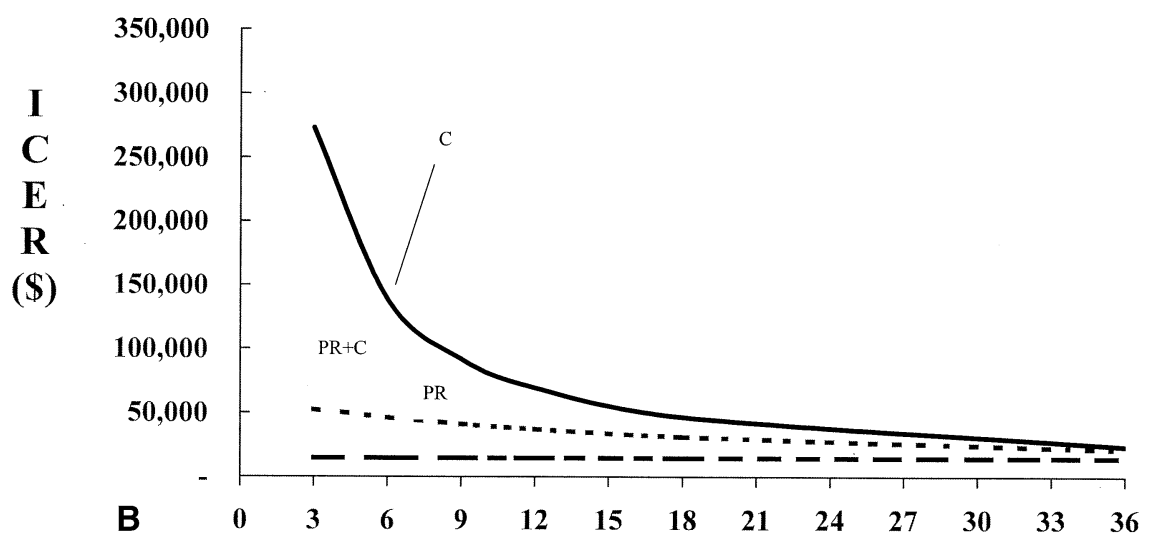

Figure 2. A, Incremental cost-effectiveness ratio (ICER) of chemotherapy alone (C), pulmonary resection alone (PR), and pulmonary resection with chemotherapy $(P R+C)$, compared with no treatments, for varying chemotherapy benefits assuming administration of 6 cycles of chemotherapy. B, ICER of $C$ alone, PR alone, and PR+C, compared with no treatment, for varying chemotherapy benefits assuming administration of 4 cycles of chemotherapy. $C_{\text {r }}$ Systemic chemotherapy.

systemic chemotherapy (6 cycles) was $\$ 99,033$ (SEM, \$9855). Compared with NoRx and assuming a 12-month survival advantage with chemotherapy, the ICER was $\$ 14,357$ per life-year gained for PR, $\$ 104,210$ per life-year gained for systemic chemotherapy, and $\$ 51,159$ per lifeyear gained for PR $+C$. Compared with PR, the ICER of $\mathrm{PR}+\mathrm{C}$ was $\$ 108,036$ per life-year gained.

Results of altering the assumed benefit of chemotherapy are displayed graphically in Figure 2. The ICER of PR remained smaller than that for $\mathrm{PR}+\mathrm{C}$, even for extremes of chemotherapy benefit (36-month survival benefit).

The outcomes and resultant ICERs for specific patient subgroups treated with PR and PR +C compared with NoRx are shown in Table 1. The result of systemic chemotherapy alone is not shown in this table because this strategy was dominated (both less effective and more costly) throughout all patient subgroups. The ICER for both PR and PR $+C$ seemed to be quite sensitive to the tumor site and disease- free interval: substantial increases in ICER were noted in patients with extremity tumors and those with a disease-free interval of less than 12 months from treatment of their primary tumor.

The effect of postresection survival on the decisionanalysis model was examined in a 1-way sensitivity analysis by using literature-based estimates of median overall survival after PR. ${ }^{3-9,11}$ The relationship between postmetastasectomy survival and the ICER for PR and PR $+\mathrm{C}$, holding costs constant, is depicted in Figure 3.

We repeated analyses under the assumption that 4 (not 6) cycles of systemic chemotherapy would be necessary for a given survival benefit. With the baseline model of a 12month survival benefit attributable to chemotherapy, the use of 4 cycles of chemotherapy resulted in an ICER of $\$ 69,497$ per life-year gained for systemic chemotherapy and $\$ 37,012$ per life-year gained for PR+C (ICER for PR was unchanged at $\$ 14,357$ per life-year gained; Figure 2). 
TABLE 1. Outcomes and resultant ICERs for PR and PR+C compared with NoRx

\begin{tabular}{|c|c|c|c|c|c|}
\hline \multirow[b]{2}{*}{ Subgroup } & \multirow{2}{*}{$\begin{array}{c}\text { NoRx } \\
\text { survival* }^{*} \\
(\mathbf{m o})\end{array}$} & \multicolumn{2}{|c|}{ PR } & \multicolumn{2}{|c|}{$\mathrm{PR}+\mathrm{C}$} \\
\hline & & $\begin{array}{c}\text { Survival }^{*} \\
(\mathbf{m o})\end{array}$ & $\begin{array}{l}\text { ICERT } \\
\text { (\$/LYG) }\end{array}$ & $\begin{array}{c}\text { Survival* } \\
\text { (mo) }\end{array}$ & $\begin{array}{l}\text { ICERT } \\
\text { (\$/LYG) }\end{array}$ \\
\hline All patients & 13 & 31 & 14,357 & 43 & 51,159 \\
\hline $\begin{array}{l}\text { Age (y) } \\
<50 \text { y }\end{array}$ & 16 & 31 & 17,310 & 43 & 57,070 \\
\hline$\geq 50 \mathrm{y}$ & 12 & 31 & 13,559 & 43 & 49,395 \\
\hline \multicolumn{6}{|l|}{ Tumor grade } \\
\hline Low & 21 & 48 & 9841 & 60 & 40,570 \\
\hline High & 13 & 27 & 18,351 & 39 & 58,949 \\
\hline \multicolumn{6}{|l|}{ Tumor site } \\
\hline Extremity/trunk & 12 & 23 & 23,245 & 35 & 66,012 \\
\hline Retroperitoneum & 12 & 56 & 6041 & 68 & 28,254 \\
\hline Visceral & 17 & 56 & 6856 & 68 & 31,277 \\
\hline \multicolumn{6}{|c|}{ Disease-free interval (mo) } \\
\hline$<12$ & 10 & 17 & 36,428 & 29 & 79,142 \\
\hline$\geq 12$ & 16 & 43 & 9724 & 55 & 40,125 \\
\hline
\end{tabular}

NoRx, No treatment; $P R$, pulmonary resection; $P R+C$, pulmonary resection and systemic chemotherapy; ICER, incremental cost-effectiveness ratio; $L Y G$, life-year gained.

*Before discounting.

tCompared with NoRx.

Finally, because the ICER of systemic chemotherapy and $\mathrm{PR}+\mathrm{C}$, compared with NoRx, was highly sensitive to changes in the assumed benefit of chemotherapy, we calculated the minimum chemotherapy benefit necessary to obtain an ICER below the conventionally accepted threshold of $\$ 50,000$ per life-year gained. With 6 cycles of chemotherapy, a greater than or equal to 32-month survival benefit must be obtained with chemotherapy to reach this threshold for systemic chemotherapy, whereas a greater than or equal to 14-month benefit must be achieved with chemotherapy for PR $+\mathrm{C}$. If only 4 cycles of chemotherapy are modeled, the required benefit of chemotherapy decreases to greater than or equal to 17 months and greater than or equal to 4 months for systemic chemotherapy and $\mathrm{PR}+\mathrm{C}$, respectively.

\section{Discussion}

The management of localized STS has improved over the past 25 years. For patients with extremity STS, there has been a migration from amputation to conservative surgery and radiotherapy as the primary form of local therapy. However, although amputation rates have declined, ${ }^{22}$ there has been no decrease in sarcoma-related mortality rates. ${ }^{23-25}$ Unfortunately, distant metastases remain a common pattern of treatment failure, ${ }^{2,26}$ and although adjuvant chemotherapy has been used to reduce this risk, its efficacy is modest at best. ${ }^{27}$ Thus, the optimal management of pulmonary metastases is a major issue for physicians treating patients with STS.

No randomized clinical trials have been performed examining whether there is a benefit to doxorubicin-based chemotherapy versus best supportive care in the treatment of patients with metastatic STS. Similarly, no clinical trials have examined whether such chemotherapy, when combined with PR, offers a survival advantage compared with operation alone. However, a cost-effectiveness analysis such as ours is dependent on a quantified survival advantage to chemotherapy; if no advantage existed, the examination of the cost-effectiveness of chemotherapy, either alone or combined with PR, would be without basis. The 12-month survival advantage with doxorubicin-based chemotherapy assumed in our base model is empiric and quite possibly represents an overestimation of the benefit of chemotherapy. Furthermore, the sensitivity analysis performed by altering the survival benefit of chemotherapy showed that a survival benefit of at least 32 months was required before the ICER with the chemotherapy-alone strategy decreased below the arbitrary, but conventionally accepted as costeffective, threshold of $\$ 50,000$ per life-year gained. The ability of any currently available chemotherapy regimen to provide such a survival advantage is not supported by any evidence, either direct or indirect.

In interpreting the aggregate data reported in this study, it is important to recognize the relative strengths and limitations of our methodology. This study used cost data (the amount of money required to actually provide a medical service) and not charge data (the amount of money a patient or third-party payer is asked to pay for a given medical service). Although charge data are generally easier to obtain, the use of cost data is less prone to the potential biases of different payer profit margins. Moreover, costs are less prone to the large variations in charges seen between dif- 
ferent institutions. Because costs theoretically do not include profits and deficits incurred by hospitals and thirdparty payers to provide a medical service, they are believed to be more reflective of the patient, physician, and societal perspectives. ${ }^{28}$ Costs used in this study (M. D. Anderson Cancer Center) may vary in other regions of the country; however, relative differences should be similar.

A potential limitation of this study is that cost and outcome estimates were not obtained from the same cohort of patients. This study took advantage of long-term outcome data from a large cohort of consecutively treated patients with STS pulmonary metastases and the only available cost data for patients with metastatic STS undergoing systemic doxorubicin-based chemotherapy or PR. The availability of cost data (rather than charges) was a consequence of specific cost accounting systems in place at the M. D. Anderson Cancer Center; such data were not available at Memorial Sloan-Kettering Cancer Center at the time of the study. In our opinion, the difficulties in using either of these patient cohorts for both cost and outcome data were far greater than the methodologic limitations of using different patient cohorts for cost and outcome data, especially because subsequent sensitivity analyses could be performed to specifically address the effects of cost and outcome variations.

Some of the 889 patients seen at Memorial Sloan-Kettering Cancer Center who did not undergo complete surgical resection of pulmonary metastases and were used to estimate the outcome of NoRx actually did receive chemotherapy at some point in their clinical course. However, because of the heterogeneity of this chemotherapy, we decided to consider them in aggregate as having received no therapy and to assign hypothetical additive benefits of chemotherapy to estimate the outcome of systemic chemotherapy. This decision may have falsely inflated the outcome of NoRx. However, such an effect would imply that the ICERs of PR reported in this study were in fact overestimates and that PR is in fact more beneficial, in terms of cost-effectiveness, than the results of this study describe.

This was a study of cost-effectiveness and not a costutility study. Otherwise stated, the units of outcome used (life-years gained) were not weighted and thus reflect only differences in survival time-not potential differences in quality of life. One could hypothesize that surgical strategies would be associated with more up-front quality of life effects, whereas the effect of systemic chemotherapy might be observed over a longer time interval, as found for other malignancies. ${ }^{29-31}$ However, the retrospective nature of this analysis did not permit assessment of temporal differences in quality of life among the different treatment strategies. Given the marked cost differences between PR and systemic chemotherapy, future prospective research in this area should include evaluation of quality-of-life parameters.

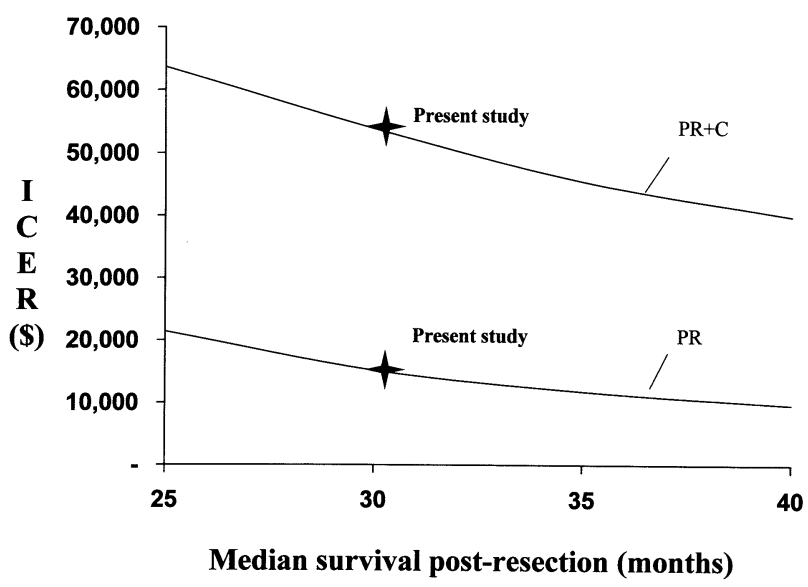

Figure 3. Effect of post-resection survival on the incremental cost-effectiveness ratio (ICER), based on literature estimates of survival, for pulmonary resection alone (PR) and PR plus chemotherapy $(\mathrm{PR}+\mathrm{C})$ compared with no treatment. Solid stars indicate the survival estimated used in this study.

This study did not estimate the baseline costs of supportive care. Thus, even though it may be reasonable to assume that the absolute costs associated with supportive care were the same for each treatment strategy, we did not account for the discounting of these costs. Discounting effectively assigns a lower value, based on an annual percentage, to costs that occur in the future compared with present-day costs. However, discounting these costs would result in lower ICERs for treatment strategies with longer survival; the lack of discounting supportive care costs therefore biased the results against $\mathrm{PR}$ and $\mathrm{PR}+\mathrm{C}$. Thus, even if we explicitly included the discounting of supportive care, it would make the strategies that included PR even more favorable from an economic perspective.

The results of this study can be applied only to patients with STS pulmonary metastases who could potentially be treated by any of the 4 outlined treatment options; decision analysis requires that a decision about treatment options can be made. Thus, we cannot extrapolate the study findings to a patient population that is ineligible for 1 or more or the 4 treatment strategies. Specifically, this study does not directly evaluate the cost-effectiveness of systemic chemotherapy in patients with STS pulmonary metastases who are not candidates for complete pulmonary metastasectomy. Such patients may represent a significant proportion of STS patients with pulmonary metastases.

Similarly, the results of this study apply to the therapeutic approaches used; significant differences in surgical or chemotherapy treatment approaches may affect cost-effectiveness. For example, the use of video-assisted thoracoscopic surgery as the surgical approach to metastasectomy may decrease the costs associated with the operation but 
may also result in poorer outcome compared with the open surgery used in this study. ${ }^{32,33}$

The results of this study provide cost-effectiveness data to support the existing therapeutic principle that when possible, pulmonary metastases should be resected. Whether the combination of resection with systemic chemotherapy is warranted, on the basis of cost-effectiveness and published outcome data, is less clear. It was not until a survival benefit attributable to systemic chemotherapy greater than or equal to 14 months was assumed that the ICER of PR $+\mathrm{C}$, compared with NoRx, decreased below the threshold of $\$ 50,000$ per life-year saved. However, compared with PR alone, the ICER of adding systemic chemotherapy required a chemotherapy survival benefit of 33 months before this threshold was reached.

In summary, this study found that, for patients with STS pulmonary metastases, PR was the most cost-effective treatment strategy evaluated; chemotherapy alone in patients with resectable STS pulmonary metastases was not a costeffective treatment strategy. Certain patient and tumor features, such as low tumor grade, nonextremity tumor location, and a disease-free interval greater than or equal to 12 months, were associated with a greater cost-effectiveness of treatment. Notwithstanding the generous assumptions about the clinical benefit of chemotherapy used in these analyses, the incremental cost-effectiveness of adding chemotherapy to PR was uncertain. It is to be hoped that randomized trials can be developed to better address the potential clinical benefit, cost-effectiveness, and quality of life associated with different treatment strategies for patients with metastatic STS.

\section{References}

1. Jemal A, Thomas A, Murray T, et al. Cancer statistics 2002. CA Cancer J Clin. 2002;52:23-47.

2. Potter DA, Glenn J, Kinsella EJ, et al. Patterns of recurrence in patients with high-grade soft-tissue sarcomas. J Clin Oncol. 1985;3:353-66.

3. Gadd MA, Casper ES, Woodruff JM, et al. Development and treatment of pulmonary metastases in adult patients with extremity soft tissue sarcoma. Ann Surg. 1993;218:705-12.

4. Billingsley KG, Burt ME, Jara E, et al. Pulmonary metastases from soft-tissue sarcoma: analysis of patterns of disease and postmetastasis survival. Ann Surg. 1999;229:602-12.

5. Jablons D, Steinberg SM, Roth J, et al. Metastasectomy for soft tissue sarcoma. J Thorac Cardiovasc Surg. 1989;97:695-705.

6. Van Geel AN, Pastorino U, Jauch KW, et al. Surgical treatment of lung metastases: the European Organization for the Research and Treatment of Cancer-Soft Tissue and Bone Sarcoma Group study of 255 patients. Cancer. 1996;77:675-82.

7. Verazin GT, Warneke JA, Driscoll DL, et al. Resection of lung metastases from soft-tissue sarcomas: a multivariate analysis. Arch Surg. 1992;127:1407-11.

8. Casson AG, Putnam JB, Natarajan G, et al. Five-year survival after pulmonary metastasectomy for adult soft tissue sarcoma. Cancer. 1992;69:662-8.

9. Vogt-Moykopf I, Krysa S, Bulzebruck H, et al. Surgery for pulmonary metastases: the Heidelberg experience. Chest Surg Clin North Am. 1994;4:85-112.
10. Robinson MH, Sheppard M, Moscovic E, et al. Lung metastasectomy in patients with soft tissue sarcoma. Br J Radiol. 1994;67:129-35.

11. Pastorino U, Buyse M, Friedel G, et al. Long-term results of lung metastasectomy: prognostic analyses based on 5206 cases. $J$ Thorac Cardiovasc Surg. 1997;113:37-49.

12. Temple LK, Brennan MF. The role of pulmonary metastasectomy in soft tissue sarcoma. Semin Thorac Cardiovasc Surg. 2002;114:35-44.

13. Van Glabbeke M, van Oosterom AT, Oosterhuis JW, et al. Prognostic factors for the outcome of chemotherapy in advanced soft tissue sarcoma: an analysis of 2,185 patients treated with anthracyclinecontaining first-line regimens-a European Organization for Research and Treatment of Cancer Soft Tissue and Bone Sarcoma Group study. J Clin Oncol. 1999;17:150-9.

14. Demetri GD, Delaney T. NCCN: sarcoma. Cancer Control. 2001;8: 94-101.

15. Mentzer SJ, Antman KH, Attinger C, et al. Selected benefits of thoracotomy and chemotherapy for sarcoma metastatic to the lung. J Surg Oncol. 1993;53:54-9.

16. Pastorino U. Metastasectomy for soft tissue sarcomas. In: Verweij J, Pinedo HM, Suit HD, editors. Soft tissue sarcomas: present achievements and future prospects. Boston: Kluwer Academic Publishers; 1997. p. 65-75.

17. Baxter G, Alvegard TA, Monge O. VIG chemotherapy in advanced soft tissue sarcoma-results of the Scandinavian Sarcoma Group SSG-X trial. Acta Orthop Scand Suppl. 1995;265:93-4.

18. Detsky AS, Krahn M, Naglie G, et al. Primer on medical decision analysis: part 2. Tree structures. Med Decis Making. 1997;17:126-35.

19. Cantor SB. Decision analysis: theory and application to medicine. Prim Care. 1995;22:261-70.

20. Tengs TO, Adams ME, Plisken JS, et al. Five-hundred life-saving interventions and their cost-effectiveness. Risk Anal. 1995;15:369-90.

21. Gold MR, Seigel JE, Russell LB, et al. Cost-effectiveness in health and medicine. New York: Oxford University Press; 1996.

22. Williard WC, Collin C, Casper ES, et al. The changing role of amputation for soft-tissue sarcoma of the extremity in adults. Surg Gynecol Obstet. 1992;175:389-96.

23. Weitz J, Antonescu CR, Brennan MF. Localized extremity soft tissue sarcoma: improved knowledge with unchanged survival over time. J Clin Oncol. 2003;15:2719-25.

24. Pisters PWT, Harrison LB, Leung JM, et al. Long-term results of a prospective randomized trial of adjuvant brachytherapy in soft tissue sarcoma. J Clin Oncol. 1996;14:859-68.

25. Rosenberg SA, Tepper J, Glatstein E, et al. The treatment of soft-tissue sarcomas of the extremities: prospective randomized evaluations of (1) limb-sparing surgery plus radiation therapy compared with amputation and (2) the role of adjuvant chemotherapy. Ann Surg. 1982;196:305-15.

26. Lewis JJ, Leung DHY, Casper ES, et al. Multifactorial analysis of long-term follow-up (more than 5 years) of primary extremity sarcoma. Arch Surg. 1999;134:199-204.

27. Tierney JF. Adjuvant chemotherapy for localized resectable soft-tissue sarcoma of adults: meta-analysis of individual data. Lancet. 1997;350: $1647-54$

28. Finkler SA. The distinction between cost and charges. Ann Intern Med. 1982;96:102-9.

29. McLeod RS. Quality of life, nutritional status and gastrointestinal hormone profile following the Whipple procedure. Ann Oncol. 1999; 10(suppl):281-4.

30. Kulaksizoglu H, Toktas G, Kulaksizoglu I, et al. When should quality of life be measured after radical cystectomy? Eur Urol. 2002;42:350-7.

31. Hillner BE, Kirkwood JM, Atkins MB, et al. Economic analysis of adjuvant interferon alfa-2b in high-risk melanoma based on projections from Eastern Cooperative Oncology Group 1684. J Clin Oncol. 1997; 15:2351-8.

32. Dowling RD, Landreneau RJ, Miller DL. Video-assisted thoracoscopic surgery for resection of lung metastases. Chest. 1998;113(1 suppl):2S$5 \mathrm{~S}$.

33. McCormack PM, Bains MS, Begg CB, et al. Role of video-assisted surgery in the treatment of pulmonary metastases: results of a prospective trial. Ann Thorac Surg. 1996;62:213-6. 